\title{
Nuevas técnicas de evaluación en el Trastorno por Déficit de Atención e Hiperactividad (TDAH)
}

\author{
Celestino Rodríguez, Paloma González-Castro, Luís Álvarez, Lorena Vicente, \\ José C. Núñez, Julio A. González-Pienda, David Álvarez, Ana Bernardo y \\ Rebeca Cerezo \\ Universidad de Oviedo (España)
}

\begin{abstract}
Este estudio pretende conocer la evolución general del proceso de diagnóstico y cuál será su futuro inmediato en el TDAH, profundizando en dos nuevas técnicas objetivas como son el EEG y el HEG. En primer lugar se pretende situar las perspectivas futuras en TDAH a corto y medio plazo. Intentando entender la realidad actual y el nuevo espectro de oportunidades que se nos abre en este sentido. En segundo lugar, específicamente se plantea el objetivo de conocer específicamente la técnica de la hemoencefalografía HEG y su antecedente inmediato EEG. Intentando mostrar sus bases biológicas, su utilidad y su accesibilidad en el diagnóstico. Así como también, completándolo con otras medidas relacionadas como puede ser el TOVA test, con una gran eficiencia y fiabilidad en el diagnóstico y seguimiento del TDAH. Todas las aportaciones teóricas, tratan de condensarse en las conclusiones aportando nuevas medidas más objetivas en el diagnóstico del TDAH, así como más sencillas de aplicar. Se discuten las implicaciones educativas de las ideas relatadas.
\end{abstract}

Palabras clave: TDAH, hemoencefalografia, HEG, EEG.

Attention Deficit/Hyperactivity Disorder (ADHD). New evaluation techniques. This study aims to determine the overall progress of the diagnostic process and what their immediate future in ADHD, digging into two new objective techniques such as the EEG and HEG. First is to put the future prospects for ADHD in short and medium term, trying to understand the current reality and the new range of opportunities open to us in this direction. Second, it specifically states the objective of knowing specifically hemoencefalografía technique nirHEG and its immediate predecessor Q-EEG, with the aim to show their biological basis, its usefulness and accessibility in the diagnosis. As well as supplementing it with other related measures such as the TOVA test, with great efficiency and reliability in the diagnosis and management of ADHD. All theoretical evidences, trying to condense the findings bring new more objective measures in the diagnosis of ADHD, as well as simpler to implement. We discuss the educational implications.

Key words: ADHD, hemoencefalography, HEG, EEG.

Correspondencia: Celestino Rodríguez. Departamento de Psicología. Área de Psicología Evolutiva y de la Educación. Facultad de Psicología. Plaza Feijoó s/n. C.P. 33003. Oviedo (España). E-mail: rodriguezcelestino@uniovi.es 
La técnica HEG (hemoencefalografía), podríamos definirla como el estudio de flujo de sangre en el cerebro. Más concretamente, es el estudio de flujo de sangre voluntariamente controlado u oxigenación en partes cerebrales expresamente escogidas. Por lo tanto, cambios fisiológicos del cerebro en activación (es decir, el cerebro activado) puede medirse por la técnica Hemoencefalografía (HEG) (Toomim y Marsh, 1999; Toomim, 2002). Esta técnica, fue validada en un estudio instruccional sobre el control voluntario del flujo sanguíneo cerebral realizado por Yoo y Jolez (2002). Se usaron estudiantes universitarios voluntarios como participantes, mostrando la mejora de determinadas áreas del cerebro con la ayuda del biofeedback por medio del FMRI (Functional Magnetic Resonance Imaging).

Esta forma de activación cerebral innova en parte, ya que se opone a la actual práctica de "bottom-up". Es decir, es contraria a las técnicas en las que se parte del movimiento para intencionalmente activar una localización anatómica del cerebro dañada. Si bien es cierto que, esta activación de una función cerebral referente a un área anatómica determinada, es posible, a menudo es ineficaz por diversas razones. Por ejemplo, el cerebro tiende a la función directa fuera de las zonas dañadas y activa algoritmos alternativos y zonas para la resolución de problemas. Por ejemplo, Taub et al. (1993) encontró que monos con infartos cerebrales no utilizan las vías neuronales rehabilitadas recientemente y que estaban implicadas como parte del control, sino que volvió a la utilización de la parte no afectada siempre que fuera posible.

En cambio, la rehabilitación de las funciones cerebrales, sostenida por la activación directa del cerebro como el caso del HEG, puede mostrar una mayor recuperación de la función normal de esas zonas dañadas (Robertson y Murre, 1999).

Las primeras investigaciones sobre las mediciones del flujo sanguíneo cerebral se iniciaron en la Unión Soviética en la década de 1960, colocándole la etiqueta de "rheoencephalography" (REG). La variabilidad en la impedancia eléctrica entre las diversas regiones en el cuero cabelludo, produce una forma de onda, a partir de la cual pueden ser derivados los componentes arteriales y venosos de la sangre. Las personas que sufren de trastornos psico-fisiológicos, lógicamente suelen tener relativamente una baja amplitud arterial en relación con su componente venoso. La técnica REG fue empleado en el diagnóstico de los trastornos circulatorios, lesiones cerebrales, la hipertensión arterial, secuelas de la exposición a la radiación y los efectos de la aceleración y los vuelos espaciales. Incluso fue utilizada para contrastar los cambios en REG con los efectos fisiológicos de la meditación y por lo tanto medirlos.

El desarrollo de esta técnica, de la medición del flujo sanguíneo, parte formalmente de estudios como el de Tokarev y Fleischman (1988), que utilizaron esta técnica de biofeedback cerebral y activación pionera REG "rheoencephalography” y lo aplicaron a los trabajadores de una fábrica soviética. Encontraron una mejora considerable en sus actividades y los índices de error se minimizaron después de varias 
aplicaciones. Estas conclusiones sugieren que, una intervención activa destinada a aumentar el flujo sanguíneo cerebral en los seres humanos es posible, así como una mejora de la función cerebral. Boynton, Engel, Glover y Heeger (1996) desarrollaron un proyecto de apoyo a estos datos en función de una relación lineal (que representan el $86.81 \%$ de la varianza) entre la respuesta FMRI y la actividad neuronal, medida sobre un pequeño volumen neuronal en un corto intervalo de tiempo.

Otra de las fuentes y antecedentes inmediatos de la técnica HEG, parte de la investigación médica en la tecnología óptica, utilizada para el seguimiento los niveles de oxígeno en el cuerpo. El ejemplo más conocido de estas técnicas en medicina, es el "oxímetro del pulso". Se ha demostrado que sus mediciones están cercanas a las mediciones por gas de las máquinas estándar de sangre en el rango de 70 a $100 \%$ de saturación de oxígeno (Williams, Mortimer y McCollum, 1996). Debido a que es rápida, no invasiva y precisa, la oximetría del pulso es utilizada rutinariamente para la vigilancia continua de los niveles de oxígeno en los pacientes durante cirugía.

Sin embargo, la oximetría del pulso solo se utiliza para medir el pulso de las arterias (Owen-Reece, Smith, Elwell y Goldstone, 1999). Al contrario, la tecnología HEG utiliza diferentes longitudes de onda de la luz, que permite la penetración más profunda en el cuerpo. Proporciona una evaluación más global de oxigenación en el tejido cerebral, especialmente en zonas específicas de la corteza cerebral.

La tecnología óptica, en la medicina, se describe usualmente como la espectroscopia infrarroja (NIRS). Elwell, Springett, Hillman y Delpy (1999) resumieron los principios físicos subyacentes NIRS y su utilidad en anestesia de adultos y neonatal de cuidados intensivos. Villringer y Chance (1997) señalaron que la NIRS puede ser especialmente útil en situaciones en las que otras tecnologías neurodiagnósticas no son factibles, por ejemplo niños con trastornos de la atención, ya que no estarían quietos en la evaluación con el resto de aparatos. Asimismo, Hock et al. (1997) predijo un papel importante de técnicas como el NIRS, especialmente para el diagnóstico o el seguimiento de trastornos del desarrollo como el caso del TDAH. Finalmente, Elwell et al. (1999) concluyó que, técnicas como el NIRS y derivadas (HEG) ofrecen la oportunidad de continuar líneas de investigación, sobre la no invasiva y cuantitativa evaluación hemodinámica y la oxigenación cerebral en las personas con problemas cerebrales. Una amplia bibliografía de la investigación NIRS está disponible en la página web del Grupo de Investigación en Óptica Biomédica de la University College, Londres.

Además es importante destacar, en relación al objetivo general de este trabajo como es el diagnóstico en el TDAH, ideas sobre cambios en el prefrontal izquierdo del cerebro del flujo de sangre como consecuencia de la concentración, descritas por Villringer, Planck, Hock, Schleinkofer y Dirnagl (1993) utilizando la tecnología NIRS. Después del establecimiento de una línea base de descanso, los sujetos fueron instruidos 
para realizar cálculos mentales durante un minuto. A los pocos segundos de esta estimulación cognitiva intencional, los niveles de hemoglobina oxigenada en sangre y aumentaron de volumen en 9 de 10 sujetos.

Con todos estos antecedentes en el panorama investigador, el americano Hershel Toomim se interesó en la tecnología de flujo sanguíneo, mientras investigaba los efectos fisiológicos del sistema biofeedback EEG. Un colega le envió una referencia de la investigación realizada por Britton Chance en espectrofotometría craneal. En 1994, mientras estudiaba el desarrollo de un dispositivo para registrar los cambios en el flujo de sangre, Toomim descubrió que aparte de esa evaluación, podría cambiar su propio nivel de hemoglobina oxigenada en la región prefrontal elegida y además hacerlo en unos pocos minutos. Él entendió la posibilidad de desarrollo de equipos de biofeedback, en lugar de utilizarlos exclusivamente para el diagnóstico y la medición de la oxigenación cerebral. Entonces nace el dispositivo que se ha denominado "nirHEG", que al igual que otras tecnologías (NIRS, REG, etc.) utiliza la luz infrarroja para medir la oxigenación a través del cráneo.

Los datos preliminares de las experiencias piloto, pusieron de manifiesto que la ubicación del sistema de retroalimentación, no la expectativa o la intención del sujeto para mantener la información en un área de percepción, determinará la ubicación de los efectos del esfuerzo. Los estudios que utilizan la función cognitiva de las diferencias entre el flujo de sangre antes y después en imágenes, mostraron que el ejercicio de activación de una determinada función cerebral, activa una serie de pequeños y dispersos volúmenes de tejido, relacionados con la función seleccionada (Posner y Raichle, 1998; Rodríguez, Fernández-Cueli, González-Castro, Álvarez y Álvarez, 2011).

Si bien el funcionamiento cerebral normal dirige cantidades locales del flujo de sangre al área funcional, la técnica nirHEG permite a un profesional psicoeducativo y al sujeto a elegir un área para realizar la activación. nirHEG permite la maximización de la oxigenación de la sangre en una posición elegida de la cabeza. El cerebro entonces aumenta el flujo sanguíneo guiado por la intención de aprovechar al máximo la información. Este enfoque, combinado con ejercicios educativos como brain-training, puede maximizar la oxigenación de la sangre directamente en determinadas áreas del cerebro.

\section{Descripción del equipo nirHEG}

La tecnología HEG utiliza la propiedad translucida del tejido biológico. El tejido biológico difunde y conduce energía radiante de muchos tipos y lo hace en una amplia gama de longitudes de onda. En concreto nirHEG usa las luces de color rojo de baja frecuencia y de infrarrojos a través de diodos emisores de luz (optodes LED). 
La fuente de luz y el receptor de luz (optode), se montan en una diadema separados entre sí por tres centímetros. Tanto la electrónica como la diadema fueron cuidadosamente diseñadas y construidas para impedir que entre luz externa y que existan fugas y que por lo tanto la luz ambiente afecte o distorsione las mediciones. Es importante destacar que, al contrario que la técnica EEG, menor grado de tensión muscular, o movimientos del sujeto de la los ojos, no afectan a la medición nirHEG. Otras posibles fuentes de error han sido investigadas y se encontró que son mínimas. Sólo alrededor del 5 al 10\% de las lecturas nirHEG provienen de la piel o tejido cráneo. Esto se debe a que estas regiones del cuerpo tienen poco flujo de sangre en relación con el tejido cerebral. Intensos movimientos corporales sí que podrían aumentar la presión arterial craneal ligeramente, pero el aplicador puede observar fácilmente estos movimientos. Por último, la calibración de la derivación del instrumento nirHEG ha resultado ser inferior al $2 \%$ en seis meses (Toomim et al., 2000).

La intensidad de penetración efectiva en el tejido cortical altamente vascular esta aproximadamente $1.5 \mathrm{~cm}$ debajo del punto medio entre la optodes. Las zonas de entrada y salida de luz están en $0,052 \mathrm{~cm} 2$ en la superficie de la piel. Los puntos de entrada y salida de luz y el de refracción y dispersión en función de las cualidades del tejido tienen una forma de plátano (Figura 1). La forma de la trayectoria óptica se discute en trabajos como Chance (1992) y Benaron et al. (1995).

Figura 1. Ilustración del arco que producen los emisores de luz en el HEG

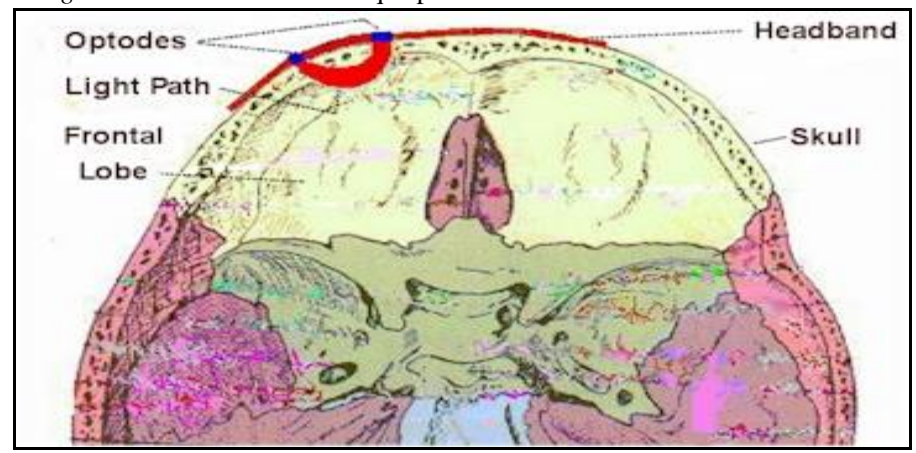

Las luces son emitidas alternativamente en la misma superficie de la piel. La emisión de luz penetra en estos tejidos y se dispersa, refractada y reflejada. Una pequeña cantidad de luz modificada por la absorción de la longitud de onda del tejido sensible se devuelve a la superficie y se mide (Jobsis, 1977; Chance, 1992). La luz roja de longitud de onda $(660 \mathrm{~nm})$ es menos absorbida por la hemoglobina oxigenada que por la hemoglobina desoxigenada. La referencia, fuente de luz infrarroja $(850 \mathrm{~nm})$, está relativamente poco afectada por el grado de oxigenación de la hemoglobina. La 
oxigenación capilar está escasamente afectada por la presión arterial periférica y está controlada principalmente por la demanda de energía de los tejidos. La concentración de hemoglobina oxigenada, por lo tanto, es una útil medida del flujo sanguíneo local. Por lo tanto, matemáticamente la fórmula de la ratio HEG, sería la siguiente

Luz roja (variable)
Ratio nirHEG= ----------
Luz infrarroja (poco afectada por la oxigenación)

La ratio o proporción nirHEG de las ondas recibidas en rojo con la luz infrarroja tiene una propiedad útil. El numerador y denominador de la relación están influenciados de la misma manera por la atenuación de la piel, el cráneo y la longitud del camino. En esta relación, por lo tanto, estas variables se desestiman. La ratio HEG es la base del entrenamiento en flujo sanguíneo. Una base normalizada para la ratio HEG se estableció mediante mediciones en FP1 en adultos, fueron 154 profesionales de asociaciones asistentes a reuniones. El valor de referencia normalizado fue establecido en $100(\mathrm{SD}=20)$; y por lo tanto, sirve para calibrar todos los nuevos espectrofotómetros. Existen diversos estudios recientes que han utilizado el sistema nirHEG en intervención y combinado con el sistema EEG, sin embargo diversos de estos estudios reclaman una validación controlada del sistema nirHEG (Mize, 2004).

Aparte de la medida de la ratio HEG, esta técnica desarrollada por el doctor Toomim, nos ofrece otras medidas interesantes, significativas y de manera inmediata.

En la pantalla además de la ratio HEG, pero dependientes de ella se nos muestran dos medidas, el segment index HEG gain, o la mejora en porcentaje de la ratio HEG en lo que llevamos de sesión, es decir en la sesión en curso. Y además nos ofrece el current index HEG gain, que es la ganancia inmediata en ese mismo momento, lógicamente este índice varía a cada segundo. Estos dos índices nos interesan desde el punto de vista de la intervención, ya que por ejemplo en TDAH, un entrenamiento productivo se refiere a unas ganancias superiores al 10-15\%. Además Toomim, no indica que podemos tener un índice de ganancia, que se define como: Ganancia = (media del segmento) / (media inicial) -1 .

Por otro lado, un índice que nos proporciona la técnica nirHEG, importante en cuanto a intervención pero también en cuanto a evaluación y destacable por lo novedoso, es el Índice de Atención (AI). Este índice AI indica que la intención de aumentar la ratio HEG presenta fallos, es decir que el sujeto es incapaz de aumentar la ratio y con ello su activación cerebral, con lo cual se puede interpretar como un lapsus de de atención y una medida de atención sostenida o concentración.

Toomim en sus estudios iniciales, utiliza este valor de AI medio como una indicación de la capacidad de concentración o una medida de atención sostenida. Resultados por encima de $87 \%$ son comunes después de los periodos de entrenamiento que el autor desarrolla en sus estudios. Sin embargo, no existen resultados publicados al respecto de este índice y sin embargo la práctica parece atribuirle grandes propiedades. Es este otro aspecto a desarrollar, una base sobre la que trabajar para conseguir una 
validación del índice, unos baremos normativos y conocer si correlaciona con otras medidas de atención sostenida, como por ejemplo el TOVA test.

\section{el TDAH.}

\section{Conclusiones, la hemoencefalografía (nirHEG) como forma de diagnóstico en}

El Trastorno por Déficit de Atención con o sin Hiperactividad (TDAH), acompañado por diversas condiciones comórbidas (oposición comportamiento, dificultades de aprendizaje, depresión/ansiedad, trastorno bipolar, tics, etc.) y otros problemas enmascarados junto al trastorno (problemas procesamiento auditivo, la depresión/ansiedad, encefalopatías, trastorno alimentarios y nutricionales, retraso mental y otros) afectan a alrededor del cinco por ciento de la población en todo el mundo (McGough y McCracken, 2000; Riccio y Reynolds, 2001) y entre el 3\% y el 6\% de niños en edad escolar (APA., 2002) y persisten en los adultos en el $40 \%$ de los casos (Fischer, Barkley, Smallish y Fletcher, 2005). Otras investigaciones, incluso aumentan esta prevalencia, situándola entre el 5 y el 10\% (Scahill y Schwab-Stone, 2000).

La complejidad del TDAH es tan heterogénea, que no puede ser caracterizado simplemente utilizando criterios conductuales, aspectos neuroanatómicos, medidas clínicas, o datos genéticos y fisiológicos exclusivamente (Biederman y Faraone, 2002; McGough y McCracken, 2000; Schweitzer et al., 2000).

Para el diagnóstico, los profesionales suelen depender de los cuestionarios provenientes de la lista de síntomas y criterios del DSM-IV, representando aspectos conductuales y sujetos a sesgos de interpretación.

Actualmente, los trabajos de resonancia magnética funcional muestran imágenes cerebrales múltiples de las área implicadas en los problemas de atención, tanto en situaciones de reposo como en situaciones de trabajo atencional y de concentración en el niño, pero sin resultados aún concluyentes (Ernst et al., 1999; Posner y Raichle, 1998; Zametkin et al., 1990).

Además, los estudios farmacológicos muestran que no existe un único modelo de la química cerebral que de cuentas de todos los casos o el rango de los síntomas en el déficit de atención (Vaidya et al., 1998). De hecho, hay una falta de acuerdo entre exactamente lo que los teóricos entienden como "déficit de atención" (Barkley, Grodzinsky y DuPaul, 1998; Mirsky, 1987, Williams y Boll, 1997; Lee, 1991).

Así pues, todo un método de tratamiento o un único modelo de evaluación no se puede esperar que pueda servir a todos los casos ya sea farmacológico, electrofisiológico, o de conducta. Del mismo modo, la medición de los resultados del tratamiento es difícil y en su mayor parte se compone de inventarios o informes subjetivos acerca del comportamiento y otras conductas superiores. Algunos médicos supervisan los progresos con ordenador basados en pruebas de rendimiento continuo, otras pruebas neuropsicológicas y algunos más avanzados incluso utilizan el Q-EEG, las exploraciones o SPECT (Sieg, Gaffnew, Preston y Hellings, 1995). Sin embargo, estos métodos no tienen clara correlación clínica estrechamente vinculada a sus datos (McGough y McCracken, 2000). El médico por lo general se queda con los inventarios y el comportamiento subjetivo como principal método de evaluación en la mejora. 
Cuestionarios de comportamiento como el EDAH, ADHD-IV, BASC, CBCL, Conners y otros muchos (McGough y McCracken, 2000) representan la base de herramientas con respecto a la cuantificación de síntomas. Estos cuestionarios y escalas, si bien son útiles, también son problemáticas porque los diferentes observadores tienden a centrarse en el comportamiento de los subgrupos de especial interés para ellos o en los ambientes de los que pueden ser especialmente conscientes. Las expectativas y la tolerancia de los observadores del comportamiento y la variación de sus opiniones pueden ser muy amplias, introduciendo una serie de sesgos en los resultados. En contraste, las pruebas de rendimiento continuo o en inglés continuous performance test $(C P T)$, son muy objetivos en su procedimiento, pero presentan un serio problema en cuanto a su acercamiento al ambiente psicoeducativo y al aula. No son aceptados, esgrimiendo que no son ecológicamente válidas. Sin embargo, otros han sido utilizados por los investigadores durante casi 50 años, por los psicólogos y psiquiatras; mucho más en los últimos diez años para cuantificar y hacer un seguimiento de los síntomas de pacientes con TDAH (Riccio y Reynolds, 2001). Hay una plétora de diferentes sistemas de CPT, que miden diferentes funciones cognitivas relacionadas con la atención. Los más comunes y los más apreciados métodos son los Conners (Conners, 1995; Barkley, 2006), el TOVA (Greenberg, 1993), el IVA (Edwards, 1998), SART, etc., en definitiva todos los que se refieren a datos normativos de la puntuación. Si bien éstos ofrecen la promesa de resultados cuantitativos y dan un "número", que dependen de estado de excitación, el esfuerzo, la calidad del sueño antes de la noche y otros factores ambientales tales como la distracción, que pueden contribuir a diferencias en el rendimiento.

Así pues, el evaluador debe interpretar los resultados cuidadosamente y valorar la episódica variación que puede no tener sentido. Muchos médicos han experimentado casos en que un niño hace poco en el CPT pero está bien clínicamente y en cambio, el niño que hace bien en el CPT, pero tiene claro deterioro clínico. En realidad, los falsos positivos, falsos negativos y las evaluaciones han sido descritas en el momento de que los CPT se utilizan de forma aislada (Trommer, Hoeppner, Lorber y Armstrong, 1988). Sin embargo, la mayoría de los niños que lo hacen mal en "la vida y la escuela" hacer mal los test de ejecución continua y los niños que lo hacen bien clínicamente, lo hacen bien en CPT (Swanson, Lerner, March y Gresham, 1999).

En el último grado de evaluación estarían las imágenes del cerebro mediante SPECT, funcional MRIs y escáner u otras tecnologías que ofrecen una gran promesa para la precisión del estado clínico, pero no son herramientas prácticas para el seguimiento de los avances y el diagnóstico. Principalmente debido a su costo, trastornos de invasión y molestias. Sin embargo, técnicas como el nirHEG, pueden ser elementos de diagnóstico y evaluación sencillos, que sustituyan de alguna forma esas técnicas de imagen de difícil acceso. Son, por lo tanto, una oportunidad única de conseguir un diagnóstico completo y contrastado.

Con estos antecedentes, merece la pena señalar las ventajas de la tecnología infrarroja sobre otros procedimientos de imágenes del cerebro como el EEG, resonancia magnética, TAC y el PET, así como también sobre las exploraciones. Estos aspectos se refieren a la objetividad presente, aun cuando el artefacto de medición de la actividad en 
los lóbulos frontales, siendo no invasiva y con un bajo costo, al alcance de cualquier proceso de diagnóstico psicológico y psicoeducativo.

\section{REFERENCIAS}

American Psychiatric Association. (2002). DSM-IV-TR. Manual diagnóstico y estadístico de los trastornos mentales. Barcelona: Masson (Edición original, 2000).

Barkley, R.A. (2006). Attention-deficit hyperactiviy disorder: A handbook for diagnosis and treatment $\left(3^{\text {rd }}\right.$ ed.); New York: Guilford.

Barkley, R.A., Grodzinsky, G.M. y DuPaul, G. (1992). Frontal lobe functions in attention deficit disorder with and without hyperactivity: A review and research report. Journal of Abnormal Child Psychology, 20, 163-188.

Benaron, D.A., Hintz, S.R., Villringer, A., Boas, D., Kleinschmidt, A., Frahm, J., et al. (2000). Noninvasive imaging of human brain using light. Journal of CerebralBlood Flow Metabolism, 20, 469-477.

Biederman, J. y Faraone, S.V. (2002). Current concepts on the neurobiology of attention deficit/hyperactivity disorder. Journal of Attention Disorders, 6 (Suppl. 1), 7-16.

Boynton, G.M., Engel, S.A., Glover, G.H. y Heeger, D.J. (1996). Linear systems analysis of functional magnetic resonance imaging in human V1. Journal of Neuroscience, 16, 4207-4221.

Chance, B. (1992). Picosecond spectroscopy and imaging with pulsed and amplitude modulated light diffused photon density waves in highly scattering media. Spie Laser Spectroscopy of Biomolocules, 1921, 2-15.

Conners, C.K. (1995). Conners' continuous performance test user's manual. Toronto, Canada: Multi-Health Systems.

Edwards, G. (1998). Determining the role of a new continuous performance test in the diagnostic evaluation of ADHD. ADHD Reports, 6(3), 11-13.

Elwell, C. y Hebden, J. (1999). Near-infrared spectroscopy biomedical optics research group. Retrieved from: $\mathrm{h} t t p: / / w w w . m e d p h y s . u c l . a c . u k /$ research/borl/research/NIR_topics/nirs.htm

Elwell, C.E., Springett, R., Hillman, E. y Delpy, D.T. (1999). Oscillations in cerebral haemodynamics. In A. Eke y D.T. Delpy (Eds.), Oxygen transport to tissue XXI (pp. 57-65). New York: Kluwer Academic/Plenum.

Ernst, M., Zametkin, A.J., Maotachik, J.A., Pascualvaca, D., Jons, P.H. y Cohen, R.M. (1999). High midbrain $[18 \mathrm{~F}]$ DOPA accumulation in children with attention deficit hyperactivity disorder. American Journal of Psychiatry, 156, 1209-1215.

Fischer, M., Barkley, R.A., Smallish, L. y Fletcher, K. (2005). Executive funcitioning in Hiperactive Children as Young Adults Attention, Inhibition, Response Perseveration, and the Impact of Comorbidity. Developmental Neuropsychology, 27(1), 107-133.

González-Castro, P., Alvarez, L., Nuñez, J.C., González-Pienda, J.A., Alvarez, D. y Muñiz, J. (2010). Cortical activation and attentional control in ADAH subtypes. International Journal of Clinical and Health Psychology, 10(1), 23-39.

Greenberg, L.M. (1993). Developmental normative data on the Test of Variables of Attention (TOVA). Journal of Child Psychology and Psychiatry and Allied Disciplines, 34, 1019-1030.

Hock, C., Villringer, K., Heekeren, H., Hofmann, M., Wenzel, R., Villringer, A., et al. (1997). A role for near infrared spectroscopy in psychiatry? In A. Villringer y U. Darnagl (Eds.), 
Optical imaging of brain function and metabolism II (pp. 105-112). New York: Plenum.

Jobsis, F.F. (1977). Noninvasive, infrared monitoring of cerebral and myocardial oxygen sufficiency and circulatory parameters. Science, 198, 1264-1267.

Lee, S.W. (1991). Biofeedback as a treatment for childhood hyperactivity: A critical review of the literature. Psychological Reports, 68, 163-192.

McGough, J.J. y McCracken, J.T. (2000). Assessment of attention deficit hyperactivity disorder: A review of recent literature. Current Opinion in Pediatrics, 12(4), 319-324.

Mirsky, A.F. (1987). Behavioral and psychophysiological markers of disordered attention. Environmental Health Perspectives, 74, 191-199.

Mize, W. (2004). Hemoencephalography-A New Therapy for Attention Deficit Hyperactivity Disorder (ADHD): Case Report. Journal of Neurotherapy, 8(3), 77-97.

Owen-Reece, H., Smith, M., Elwell, C.E. y Goldstone, J.C. (1999). Near infrared spectroscopy. British Journal of Anesthesia, 82, 418-426.

Posner, M.I. y Raichle, M. (1998). The neuroimaging of human brain function. PNASOnline, 95(3), 763-764.

Riccio, C.A. y Reynolds, C.R. (2001). Continuous performance tests are sensitive to ADHD in adults but lack specificity: A review and critique for differential diagnosis. Annals of the New York Academy of Sciences, 931, 113-139.

Robertson, I.H. y Murre, J.M.J. (1999). Rehabilitation of brain damage: Brain plasticity and principles of guided recovery. Psychological Bulletin, 125(5), 544-575.

Rodríguez, C., Fernández-Cueli, M., González-Castro, P., Álvarez, L. y Álvarez, D. (2011). Diferencias en la fluidez sanguínea cortical en los subtipos de TDAH. Un estudio piloto. Aula Abierta, 39(1), 25-36.

Scahill, L. y Schwab-Stone, M. (2000). Epidemiology of ADHD in School-age children. Child and Adolescent Psychiatric Clinics of North America, 9, 501-555.

Schweitzer, J.B., Faber, T.L., Grafton, S.T., Tune, L.E., Hoffman, J.M. y Kilts, D.C. (2000). Alterations in the functional anatomy of working memory in adult attention deficit hyperactivity disorder. American Journal of Psychiatry, 157, 278-280.

Sieg, K.G., Gaffnew, G.R., Preston, D.F. y Hellings, J.A. (1995). SPECT brain imaging abnormalities in attention deficit hyperactivity disorder. Clinical Nuclear Medicine, 20, 55-60.

Swanson, J., Lerner, M., March, J. y Gresham, M. (1999). Assessment and intervention for attention-deficit/hyperactivity disorder in the schools: Lessons from the MTA study. Pediatric Clinics of North America, 46, 993-1009.

Taub, E., Miller, N.E., Novack, T.A., Cook, E.W., Fleming, W.C., Nepomuceno, C.S., et al. (1993). Technique to improve chronic motor deficit after stroke. Archives of Physical Medicine and Rehabilitation, 74, 347-354.

Tokarev, V. y Fleishman, A. (1988). Technical and engineering implementation of REG (Rheoencephalography) biofeedback training at the West Siberian Metallurgical Plant. Russian Academy of Medical Sciences, 23, 265-345.

Toomim, H., Mize, W., Yeekwong, P., Toomim, M., Marsh, H., Kozlowski,G.P., et al. (2004). Intentional increase of cerebral blood oxygenation using Hemoencephalography: An efficient brain exercise therapy. Journal of Neurotherapy, 8(3), 5-21.

Toomim, H. (2002). Neurofeedback with Hemoencephalography (HEG). Explore! Forthe Professional, 11(2), 19-21.

Toomim, H. y Marsh, R. (1999). Biofeedback of Human Central Nervous System ActivityUsing Radiation Detection. Washington, DC: US Patent and Trademark office.US Patent number 5,995,857. 
Toomim, H., Remond, A., Toomim, M., Marsh, R., Kozlowski, G., Kimble, M., et al. (2000, September). Intentional increase of cerebral blood oxygenation: A brain exercise therapy. Paper presented at the annual conference of the Society for Neuronal Regulation, St. Paul, MN.

Trommer, B.L., Hoeppner, J.B., Lorber, R. y Armstrong, K. (1989). Comment in: Journal of Development and Behavioral Pediatrics, 10(5), 284-286, on Pitfalls in the use of a continuous performance test as a diagnostic tool in attention deficit disorder.

Villringer, A. y Chance, B. (1997). Non-invasive optical spectroscopy and imaging of human brain function. Trends in Neuroscience, 20, 435-442.

Villringer, A., Planck, J., Hock, C., Schleinkofer, L.,y Dirnagl, U. (1993). Near infrared spectroscopy (NIRS): A new tool to study hemodynamic changes during activation of brain function in human adults. Neuroscience Letters, 154, 101-104.

Williams, M.A. y Boll, T.J. (1997). Recent advances in neuropsychological assessment of children. In G. Goldstein y T.M. Incagnoli (Eds.), Contemporary approaches to neuropsychological assessment (pp. 221-267). New York: Plenum.

Williams, I.M., Mortimer, A.J. y McCollum, C.N. (1996). Recent developments in cerebral monitoring-Near-infrared light spectroscopy. An overview. European Journal of Vascular and Endovascular Surgery, 12, 263-271.

Yoo, S.S. y Jolez, F.A. (2002). Functional MRI for neurofeedback: Feasibility study on a hand motor task. Neuroreport, 13(11), 1377-1381.

Zametkin, A.J., Nordahl, T.E., Gross, M., King, C., Semple,W.E., Rumsey, J., et al. (1990). Cerebral glucose metabolism in adults with hyperactivity with childhood onset. New England Journal of Medicine, 323, 1361-1366. 